MODELING, IDENTIFICATION AND CONTROL, 1987, VOL. 8, NO. 2, 69-89

doi:10.4173/mic.1987.21

\title{
Pressurized water reactor control by the hierarchical method
}

\author{
ILKKA LEIKKONEN†
}

Keywords: Pressurized water reactor, optimal control, power distribution, load cycle

A simple version of the hierarchical optimization method is used to solve the control problem for the power distribution of a pressurized water reactor. The control period is about twenty hours. The control objectives include the total power, power distribution and use of boron. The controllers are a rod bank, soluble boron in the coolant and the coolant temperature deviation. A onedimensional non-linear core model is used, with full xenon-iodine dynamics.

\section{Introduction}

The power of a pressurized water reactor (PWR) is controlled with control rods and soluble boron in the cooling water. The control rods are inserted from the top of the core. The further they are inserted, the more neutrons are absorbed and as a consequence the power is reduced. Similarly, increasing the boron concentration also reduces the power. The possibility to control the power by varying the coolant temperature has been studied lately (see for example, Mayer et al. 1982). This produces an indirect effect: when the temperature is lowered, the neutrons are better moderated and thus the power is increased, and vice versa.

Nuclear power reactors are at present normally run at constant, full power. Load cycling is only practised in some cases. This means that low power is produced at night and during weekends, when the power demand is low, and full power during the day (Gautier 1985). It might be expected that such load cycling will become more common, if the share of nuclear power in electricity production increases (which may not now happen).

\subsection{Control problem}

If the reactor power is changed regularly, this raises the question of which is the best or most optimal way of controlling the power. The optimal control problem has been reviewed by Ebert (1982). Even at constant full power some control problems may appear. One important example is that of preventing or suppressing the so-called xenon-induced power oscillations (Schott 1984, Schulz and Lee 1980), which can result from the interaction of the fission product xenon-135 and the neutron flux.

Changing the boron concentration in the cooling water changes the power in the core homogeneously. The reduction of the boron concentration is accomplished by pumping clean water into the cooling circuit, and removing a corresponding amount of cooling water. This operation produces radioactive waste water, which must be treated at some cost. It is of interest to find ways of reducing the changes in boron concentration.

Received 15 December 1986

† OECD Halden Reactor Project, P.O. Box 173, N-1751 Halden, Norway. 
The control rods affect the power more locally, changing the power distribution as well as total power. There are typically several dozen control rods (around sixty). Several rods are connected to rod banks. The rods in one bank move together. There are different strategies to operate the rods. At or near full power it is common to have all rod banks out (or almost out) of the core, except for one bank. This is used to control the power. Thus, there are two control elements available, the boron concentration and the position of the single rod bank which is active.

The coolant temperature can be changed by a mismatch between the reactor power and cooling power. The changing temperature affects the total reactor power, and the power distribution slightly. Thus it can be used instead of, or in addition to, boron which gives a third controller. This can be of importance especially when approaching the time for fuel reload, when the fuel is almost consumed, and consequently the boron concentration is small.

In a core with fresh fuel where the amount of uranium atoms is large and the amount of neutron-absorbing fission products is small, the boron concentration must be large, in order to compensate for the large reactivity of the fresh fuel. When the fuel is consumed, 'burned', the amount of uranium atoms is reduced and the amount of neutron absorbing fission products is increased. Thus the boron concentration must be gradually reduced due to the reduced reactivity of the fuel. Though the boron concentration finally approaches zero, the temperature changes are limited, due to technical, economic and safety reasons.

\subsection{Control objectives}

It is important that the reactor produces the power which is required. It is also important that the power distribution in the core is near a desired distribution, which can be flat, i.e. the power density in all parts of the core is about the same. The desired power distribution can also be rounder, which results in smaller losses of neutrons to the surroundings, bccause the power density at the outer parts of the core is small. This is significant for cores containing used fuel.

The power density in the core must always be limited in some way. Because it is difficult to measure and directly control the local power density, some indirect means must be used to limit it. The so-called axial offset is a rough measure of the power density distribution. It is defined as the difference in the power produced in the lower and upper halves of the core, divided by the total power. If the axial offset (which is easy to measure) is limited, then the power density is also limited, and it is improbable (but not inconceivable) that there were strong peaks in the power density. The limits on axial offset can depend on the total power, with tighter limits at full power, and more relaxed limits at partial power. It can be argued that with improved control and better surveillance of the core these limits might be relaxed, which would result in more economical use of fuel.

The control problem can be formulated in the following manner: Given the total power requirement for the next twelve ... twenty-four hours, find how to operate the available controllers in a way that is best, or optimal in some sense. Optimality refers to a good balance between the different aspects of the problem, such as: desired power, desired power distribution, small changes in the boron concentration, restricted changes in the coolant temperature. Furthermore, the different limits, or constraints on power density, boron concentration, coolant temperature and the control rod position must be taken into account. 


\subsection{Additional aspects}

In order to be able to control the core in an optimal way, its existing state and properties must be known. The state involves the unmeasurable concentrations of the fission products xenon-135 and iodine-135, and the measurable power density. The properties include different cross sections, feedback coefficients etc. This means that a core surveillance system must be available, which includes at least an accurate core model and means (program) for deriving the unmeasurable variables and parameters from the measurements, using the core model. An accurate core model is also important for the optimal control itself, for checking results and calculating different parameters. In this way an optimal control 'package' is only a part of an extensive core surveillance system (Berg et al. 1984).

\section{Core dynamics}

The accurate description of the behaviour of a PWR core requires a complicated model with several thousand variables. For control purposes a simpler model can be expected to suffice, provided that it retains the essential features of the core dynamics.

The dynamics of a PWR core can be divided into three different time scales: the behaviour of neutrons with time constants of one minute or less, the behaviour of the fission products xenon- 135 and iodine- 135 with time constants of hours, and the gradual changes in the properties of the core, due to the consumption of fuel.

The dynamics (and difficulties) of the control problem discussed here are determined by the behaviour of xenon and iodine. The behaviour of neutrons (neutronics) can be described using equilibrium equations, because of the small time constants involved. The changes in the core properties are so slow that they can be considered to be constant during the control period of one day or less.

\subsection{Neutronics}

At steady state the production of neutrons in fission and losses due to scattering (out of core, to lower energies, or out of the volume in consideration) and absorption are in balance. Though almost the same expression would be obtained as (1) from the one-group method, the 1.5 group diffusion equation has been used, this can be expressed as (Silvennoinen 1976):

$$
-D \nabla^{2} \Phi+\Sigma_{r} \Phi=k \Sigma_{A} \Phi
$$

where

$D$ is the diffusion coefficient

$\Phi$ is the fast neutron flux (neutrons $/ \mathrm{cm}^{2} \mathrm{~s}$ )

$\Sigma_{r}$ is the neutron removal cross section

$k$ is the infinite multiplication factor (ratio of neutrons produced to neutrons absorbed)

$\Sigma_{A}$ is the absorption cross section

Equation (1) states that the diffusion of neutrons to and from the volume considered, neutron removal and neutron production are in balance. The boundary condition is that the neutron flux is zero slightly outside of the core (other formulations can also be used). 
The parameters of eqn. (1), notably the cross sections and the infinite multiplication factor depend on the core properties and controllers. It is important for the core dynamics that they also depend on the concentration of the fission product xenon-135, which has a large absorption cross section for neutrons. Further, the parameters depend on the neutron flux through the power density. This results in feedback to the neutron flux. In pressurized water reactors this feedback is negative. This means that after a disturbance the neutron flux (and thus power) adjusts to a form and level where eqn. (1) holds.

Equation (1) is discretized for numerical solution by dividing the core into a number of nodes, or volume elements, and transforming eqn. (1) to a form which describes the neutron balance between these nodes. The power density in the nodes can be used instead of the neutron flux, because the power density depends directly on the neutron flux. For a control problem this new form of eqn. (1) will be a constraint that binds the power densities (or neutron flux) in the nodes together, and tells how the controllers affect the power density.

\subsection{Xenon-iodine dynamics}

Xenon-135 is a fission product that has a very large absorption cross section for neutrons. The absorption of a neutron leads to xenon-136, which has a small absorption cross section. Xenon-135 is produced partly directly in fission, partly as a decay product of iodine-135, which can be considered as a direct fission product, because the half life of the precursor is short. (In the following, the mass number 135 is dropped.) Xenon is unstable, with a half life of about nine hours. Thus it is removed by radioactive decay, and neutron absorption (or 'burning' of the xenon). The equations governing the production and decay of xenon and iodine are (Glasstone and Sesonske 1967):

$$
\begin{gathered}
\frac{d X}{d t}=\frac{\gamma_{\mathrm{x}}}{\mathrm{E}} P-\lambda_{\mathrm{x}} X-\frac{\Gamma}{\mathrm{E}} P X+\lambda_{I} I \\
\frac{d I}{d t}=\frac{\gamma_{I}}{\mathrm{E}} P-\lambda_{I} I
\end{gathered}
$$

where

$$
\begin{array}{ll}
\left.\Gamma=\sigma_{x} / \varepsilon \Sigma_{f} \text { (about } 3 \times 10^{-17} \mathrm{~cm}^{3}\right) \\
\sigma_{x} \text { microscopic absorption cross section of xenon } \\
\varepsilon \quad \text { fast fission factor }(\approx 1) \\
\Sigma_{f} \text { fission cross section }
\end{array}
$$

and

$\gamma_{\mathrm{x}}, \gamma_{\mathrm{I}}$ fission yield of xenon and iodine $(\approx 0.003,0.062)$

$\lambda_{\mathrm{x}}, \lambda_{\mathrm{I}}$ decay constants of xenon, iodine $\left(2 \cdot 1 \times 10^{-5} / \mathrm{s}, 2.87 \times 10^{-5} / \mathrm{s}\right)$

E energy per fission $\left(3 \cdot 2 \times 10^{-11} \mathrm{~J}\right)$

$X, I$ xenon and iodine concentrations (atoms $/ \mathrm{cm}^{3}$ )

$P \quad$ power density $\left(\mathrm{W} / \mathrm{cm}^{3}\right)$

In eqn. (2), the neutron flux is replaced by the power density. The power density is directly proportional to the neutron flux. In the discussion of core control it is more natural to use the power density directly, because it is the variable to be controlled. 
In the xenon-iodine equations, eqn. (2), the power density appears explicitly, and in the neutron balance equation, eqn. (1), the parameters depend on the xenon concentration. Thus the power density (or neutron flux) and the xenon concentration interact rather strongly, so that the dynamics of a pressurized water reactor core are determined to a great degree by this interaction. In some cases this interaction can lead to what are called xenon oscillations, in which the local power density, and the xenon and iodine concentrations oscillate. This is undesirable, and the power in the core must be controlled so that such oscillations are not excited and any existing oscillations are suppressed.

When the core is divided into nodes for numerical solution, the xenon-iodine dynamics eqn. (2) must be satisfied (and solved) in cach node. These equations are coupled through the power density, because the neutron balance equation, eqn. (1) couples the power densities in the nodes together.

\section{Core model}

\subsection{Neutronics model}

Because the power density in the core is proportional to the fission rate, which again is proportional to the neutron flux, the core model used in these control studies uses the power density instead of the neutron flux. A one-dimensional discrete core model is used. It can be derived by considering the neutron balance between the nodes directly, leading to the use of coupling coefficients between the nodes.

These coupling coefficients can be obtained from the neutron balance eqn. (1) (Silvennoinen 1976, Locke and Blobel 1974). After some further simplifications a one-dimensional ('FLARE' type) nodal model results, describing the neutron balance between the vertical nodes, using power densities in the nodes. For each node $i$ the balance can be written as

$$
\boldsymbol{P}_{i}=k_{i} \omega_{i i} \boldsymbol{P}_{i}+k_{i} P_{i-1} \omega_{i, i-1}+k_{i} P_{i+1} \omega_{i, i+1}
$$

where

$P$ is the power density

$k$ is the infinite multiplication factor

$\omega$ are the coupling coefficients

$k$ depends on the cross sections and other parameters of eqn. (1). Index $i$ refers to node $i, i+1$ and $i-1$ to nodes below and above node $i$, respectively. Only nodes with core material are included in eqn. (3). Thus for the topmost node $(i=1)$ the second term and for the bottom node $(i=N)$ the last term on the right hand side of eqn. (3) is dropped.

The coupling coefficients are $(j=i, i+1$, or $i-1)$

$$
\omega_{i j}=\frac{M_{i j}\left(k_{j}-k_{i}\right)}{4 h k_{j}}+\frac{M_{i j}^{2}\left(k_{j}+k_{i}\right)}{2 h^{2} k_{j}} \quad \text { for } i \neq j
$$

and

$$
\omega_{i i}=1-\omega_{i+1, i}-\omega_{i-1, i} \quad \text { for } i=j
$$


For the nodes adjacent to the reflector $(i=1$ and $i=N)$ the coupling coefficient to the reflector is needed for use in eqn. (5). It is (subscript $\mathrm{R}$ refers to reflector)

$$
\omega_{\mathrm{Ri}}=\frac{2 M_{\mathrm{iR}}^{2}}{h k_{i}(h+2 \lambda)}
$$

In these equations

$k$ is the infinite multiplication factor

$M$ is the migration length $\left(M^{2}=D / \Sigma_{r}\right.$ from eqn. (1))

$h$ is the node spacing

$\lambda$ is the so called extrapolation length (or reflector saving, typically about $25 \mathrm{~cm})$

In the model all the other parameters of eqns. (4)-(6) are assumed to be constant, except for the infinite multiplication factor. It is assumed to depend linearly on the power density (this gives a power feedback), the boron concentration, control rod position and the coolant temperature deviation. The infinite multiplication factor of node $i$ is (index $i$ omitted)

$$
k=k_{0}\left[1-a_{1}\left(P-P_{0}\right)-a_{2}\left(X-X_{0}\right)-a_{3}\left(B-B_{0}\right)-a_{4} C-a_{5} T\right]
$$

where

$k$ is the infinite multiplication factor

$P$ is the power density

$X$ is the xenon concentration

$B$ is the boron concentration in the coolant

$C$ is the control rod fraction in node $i, 0 \leqslant C \leqslant 1$

$T$ is the temperature deviation from normal valuc

$a_{1}, a_{2}, a_{3}, a_{4}, a_{5}$ are coefficients, that can be different in each node.

subscript 0 refers to a reference state

This core model is used in two ways. Firstly it is considered to be a constraint in the optimal control problem and secondly, it is used to calculate the power density in the nodes for known controller positions (boron, rod, temperature) and the xenon density.

In the latter case eqns. (3) for all nodes, together with eqn. (7) form a set of non-linear equations, which are solved iteratively by Newton's method. (It should be noted that traditionally an equation such as eqn. (3) is considered to be an eigenvalue equation, and is solved accordingly. However a faster convergence is obtained here when it is treated as a non-linear equation.)

It is clear that such a simple one-dimensional model does not describe the core accurately. In order to improve the model, i.e. the agreement with more accurate models, some additional non-linearities are included approximately in the model. These adjustments are only used, when eqns. (3) and (7) are employed as a model, but not when they are used as constraints in the optimal control problem, for simplicity. The included nonlinearities are:

$k_{0}$ in eqn. (7) depends on the average power density (or coolant temperature)

coefficient $a_{3}$ for boron in eqn. (7) depends on the average power density

a term proportional to the product of controller fraction and xenon concentration is added to eqn. (7) 
(in addition parameter $\Gamma$ in eqn. (9) (next section) depends on the average power density)

After these additions and modifications the performance of the simple onedimensional core model is satisfactory, when compared with a more accurate model. Such an accurate (three-dimensional) core model is needed in the control studies discussed here. It is used to generate the parameters for the simpler model, to give an initial state and check the results from optimal control calculations. (In fact, it could also be used to calculate the power densities, instead of the simple model, but this has not been attempted.) The CYGNUS core model (Hval 1982) is used here as the more accurate model.

\subsection{Linear model}

In the solution of the optimal control problem a linear model is needed. It can be different at each time step. This gives the change in the power density as a function of the changes in the boron concentration, the control rod position and the temperature deviation, as (for derivation see Haugset and Leikkonen 1980):

$$
\Delta P=M_{\mathrm{b}} \Delta B+M_{r} \Delta R+M_{t} \Delta T
$$

where

$\Delta \boldsymbol{P}$ is a vector giving the change in power density in the nodes

$M$ with subscripts $b, r, t$ are the coefficients (vectors) of the linear model

$\Delta B, \Delta R, \Delta T$ are the deviations in the boron concentration, control rod position and coolant temperature, respectively, from the reference state values

Note that the linear model is only used as a tool in the solution process, the optimal control problem is still formulated with the non-linear model of eqn. (3).

\subsection{Discrete xenon-iodine equations}

In the control problem discussed here, time is discretized, with time steps of about half an hour to an hour. Thus the xenon-iodine dynamics must be expressed in discrete, solved form. This means that the xenon and iodine concentrations at time step $s+1$ are expressed as functions of concentrations at time step $s$, and of power density at time step $s$ and $s+1$. This is needed for all nodes.

In order to solve eqn. (2) the non-linear term $P(t) X(t)$ is approximated (with a normally negligible error) over time interval $\left[t_{0}, t_{1}\right]$, i.e. over one time step, as

$$
P(t) X(t) \approx X(t)\left[P\left(t_{1}\right)+P\left(t_{0}\right)\right] / 2+X\left(t_{0}\right)\left\{P(t)-\left[P\left(t_{1}\right)+P\left(t_{0}\right)\right] / 2\right\}
$$

Further, it is assumed that the power density $P$ changes linearly with time from $P\left(t_{0}\right)$ to $P\left(t_{1}\right)$. The power density at time $t_{1}$ may not be known, because it depends on the xenon concentration at that time. With these approximations an analytical solution is obtained, containing some exponentials. When the exponentials are approximated with a second order expansion, the desired discrete solution is 
obtained. For xenon it is (to be used for all nodes $i$, index $i$ is omitted):

$$
\begin{aligned}
X(s+1)= & X(s) D_{X 0}+I(s) D_{I 0}+P(s) D_{P 0}+P(s+1) D_{P 1} \\
& +X(s) P(s) D_{X P 0}+X(s) P(s+1) D_{X P 1} \\
& +P^{2}(s) D_{P 20}+P(s) P(s+1) D_{P P} \\
& +P(s) I(s) D_{P I 0}+P(s+1) I(s) D_{P 11} \\
& +X(s) P^{2}(s) D_{X P 2}+X(s) P(s) P(s+1) D_{X P P}
\end{aligned}
$$

where

$$
\begin{aligned}
& D_{X 0}=1-\lambda_{\mathrm{x}} \Delta t+\lambda_{\mathrm{x}}^{2} \Delta t^{2} / 2 \\
& D_{P 20}=-\Gamma \gamma_{\mathrm{x}} \Delta t^{2} / 4 \mathrm{E}^{2} \\
& D_{I 0}=\lambda_{I} \Delta t-\lambda_{\mathrm{I}} \Delta t^{2}\left(\lambda_{\mathrm{I}}+\lambda_{\mathrm{x}}\right) / 2 \\
& D_{P P}=-\Gamma \gamma_{\mathrm{x}} \Delta t^{2} / 4 \mathrm{E}^{2} \\
& D_{P 0}=\left(\gamma_{\mathrm{x}} \Delta t-\gamma_{\mathrm{x}} \lambda_{\mathrm{x}} \Delta t^{2}+\gamma_{\mathrm{I}} \lambda_{\mathrm{I}} \Delta t^{2}\right) / 2 \mathrm{E} \\
& D_{P 1}=\gamma_{\mathrm{x}} \Delta t / 2 \mathrm{E} \\
& D_{X P 0}=\left(\Gamma \lambda_{\mathrm{x}} 3 \Delta t^{2} / 2-\Gamma \Delta t\right) / 2 \mathrm{E} \\
& D_{P I O}=-\Gamma \lambda_{\mathrm{I}} \Delta t^{2} / 4 \mathrm{E} \\
& D_{P I 1}=-\Gamma \lambda_{1} \Delta t^{2} / 4 \mathrm{E} \\
& D_{X P 1}=\left(-\Gamma \Delta t+\Gamma \lambda_{x} \Delta t^{2} / 2\right) / 2 \mathrm{E} \\
& D_{X P 2}=\Gamma^{2} \Delta t^{2} / 4 \mathrm{E}^{2} \\
& D_{X P P}=\Gamma^{2} \Delta t^{2} / 4 \mathrm{E}^{2}
\end{aligned}
$$

The solution for iodine is

$$
I(s+1)=I(s) A_{I 0}+P(s) A_{P 0}+P(s+1) A_{P 1}
$$

where

$$
\begin{aligned}
& A_{I 0}=1-\lambda_{1} \Delta t+\lambda^{2} \Delta t^{2} / 2 \\
& A_{P 0}=\left(\gamma_{1} \Delta t-\gamma_{1} \lambda_{1} \Delta t^{2}\right) / 2 \mathrm{E} \\
& A_{P 1}=\gamma_{1} \Delta t / 2 \mathrm{E}
\end{aligned}
$$

$\Delta t$ is the time step length

\section{The optimal control problem}

The problem to be solved is that of finding the 'best', or optimal control over what is typically a period of about 20 hours. 'Control' means here the control rod movements, changes in the boron concentration and the coolant temperature deviation during the defined period. The goal is to keep the average power density near the required values, keep the power density distribution close to the required distribution and to only have small changes in the boron concentration. In addition, the control and core state can be subject to some constraints. It is assumed that the initial state is known and that the desired average power density (i.e. the total reactor power) is defined. Possible inaccuracies, disturbances, noise etc. are not considered, the control problem is thus deterministic.

Mathematically the problem is formulated using an objective function, which is to be minimized. The objective function contains terms that describe the goals of the control. If a small value is obtained for the objective function, the control can be considered to be rather good. In this case the function is

$$
\begin{array}{r}
2 J=\sum_{s} W_{P}\left\{\frac{1}{N}\left[\sum_{i} P_{i}(s)\right]-P_{d}(s)\right\}^{2}+\sum_{s} \sum_{i} W_{D}\left[P_{i}(s)-P_{d i}(s)\right]^{2}+ \\
\sum_{s} W_{B}[B(s)-B(s-1)]^{2}
\end{array}
$$


where

$J \quad$ is the objective function

$W_{P}, W_{D}, W_{B}$ are weights

$P_{i}(s)$ is the power density in node $i$ at time step $s$

$P_{d}(s)$ is the required average power density

$P_{d i}(s)$ is the required power density in node $i$

$B(s)$ is the boron concentration

$N$ is the number of nodes, all are of equal size

The first term in eqn. (11) describes the deviation from the required power, the second is the deviation from the required power distribution, and the last term is a measure of the changes in the boron concentration. The sums with index $s$ are taken over all time steps, and sums with index $i$ are over all nodes. The weights could be time dependent, but here it is assumed for simplicity that they are constants.

The control can be subject to some constraints. The control rod insertion is always constrained to be within the core, i.e.

$$
0 \leqslant R(s) \leqslant N h
$$

where

$R(s)$ is the control rod insertion at time step $s$

$N$ is the number of nodes

$h \quad$ is the node height (or node spacing).

The coolant temperature deviation is also limited,

$$
-T_{0} \leqslant T(s) \leqslant T_{0}
$$

where $T_{0}$ is a suitable limit, around $5-10^{\circ} \mathrm{C}$. The limits need not be symmetric, here they are the same in order to simplify matters.

The power density distribution can be constrained so that the difference of power produced in the lower and upper halves of the core must be smaller than a given fraction of the total power (limited power axial offset), i.e.

$$
-\Lambda_{0} \sum_{1}^{N} P_{i}(s) \leqslant-\sum_{1}^{N / 2} P_{i}(s)+\sum_{N / 2+1}^{N} P_{i}(s) \leqslant \Lambda_{0} \sum_{1}^{N} P_{i}(s)
$$

where $\Lambda_{0}$ is a suitable limit, about $0 \cdot 05-0 \cdot 1$. Other notation is as in the previous equations. The number of nodes $(N)$ is assumed to be even, for the sake of simplicity. The upper and lower limits are again symmetric and independent of time just for convenience.

In practice the rate of change of the boron concentration is limited by technical reasons. However, the attempt to incorporate a constraint in the solution method described here met some problems. Thus the rate of change is only included in the objective function (the last term in eqn. (11)).

Although not constraints in practical meaning of the term, eqns. (3) (for all nodes) together with eqn. (7), and eqns. (9) and (10), defining the behaviour of the core, are mathematically taken as constraints for the control problem. The optimal control problem is thus formulated as a rather normal function minimization, subject to some constraints in the following way: 
For all time steps $s=1, \ldots, K$ the boron concentration $B(s)$, control rod position $R(s)$ and coolant temperature deviation $T(s)$ must be found that minimize the objective function $J$ of eqn. (11). In the minimization the constraints defined with eqns. (3), (7), (9), (10), (12), (13) and (14) must be taken into account.

A problem like this is normally solved by the help of the Lagrange coefficients (or using optimal control theory, which in this case means about the same). This leads to a large set of non-linear equations. Although in this relatively simple case the set of equations might be solvable by normal means, a similar problem with a more detailed core model and perhaps more control rods might not. Thus it was decided that other solution methods should be attempted. Beraha and Karppinen (1981) have applied the hierarchical solution method to a similar control problem, and it was decided to adopt a simple version of that method to the present control problem.

\section{The hierarchical formulation}

The hierarchical method is a special iterative method, see for example (Singh and Titli 1978). The problem to be solved is divided into several smaller sub-problems, containing some extra parameters for coupling between the sub-problems. The solution is accomplished on two (or more) levels. On the lowest level these sub-problems are solved independently. On the higher level(s) the sub-problems are modified, or coordinated with help of those extra parameters in such a way, that the solution of the sub-problems approach the solution of the total problem.

The present optimal control problem is solved using the hierarchical method by dividing it into two sub-systems, each with their own power densities and xenon concentrations. On one sub-system the power density is controlled with the proper controllers (boron, control rod, coolant temperature). In the other system the power density itself is treated as the controller. For the converged solution these two power densities have the same values.

The transformation to the hierarchical form is done as follows. First new, calculational power densities, denoted by $\Pi(s)$ and xenon concentrations, denoted by $\Psi(s)$ are introduced, together with the constraints that these are actually the same as, the proper power densities $\boldsymbol{P}(s)$ and the xenon concentrations $\boldsymbol{X}(s)$, respectively. In componentwise equation form these constraints can be written as

$$
\Pi_{i}(s)=P_{i}(s)
$$

and

$$
\Psi_{i}(s)=X_{i}(s)
$$

The first constraint is relaxed during the solution process, however.

The new power density $\boldsymbol{\Pi}(s)$ is substituted for the proper power density $\boldsymbol{P}(s)$ in the equations for xenon-iodine dynamics. eqns. (9) and (10), and in the objective function. The new xenon concentration is used in the equations for the neutronics, or actually in the equation for the infinite multiplication factor $k$, eqn. (7).

Next, the Lagrange function is formed for this 'new' optimization problem, in the usual way. The objective function, eqn. (11) (with $\Pi$ instead of $P$ ) is augmented with the equations ('constraints') for the neutronics (eqn. (3)) and xenon-iodine dynamics (again with $\Pi$ instead of $P$, eqns. (9) and (10)), multiplied by the Lagrange coefficients $Q(s), \boldsymbol{Y}(s)$ and $\boldsymbol{H}(s)$, for power, xenon and iodine equations, respectively. 
Further, the new constraints (eqns. 15 and 16) connected to the new variables are multiplied by the Lagrange coefficients $\alpha(s)$ and $\gamma(s)$, respectively and added to the Lagrange function. (All the Lagrange coefficients are vectors, with $N$ elements. $N$ is the number of nodes in the core model. Thus the multiplications are actually vector operations.) The 'proper' constraints for the control rod position, the coolant temperature and the power density, eqns. (12), (13) and (14) are not yet included.

Finally, this Lagrange function for the total problem is divided into the two sub-systems. The first, called the power sub-system, obtains the terms where the proper power density $\boldsymbol{P}(s)$ and the new xenon density $\Psi(s)$ appear together with the boron term from the objective function. The Lagrange function for the power subsystem is thus

$$
\begin{aligned}
L_{1}= & \frac{W_{B}}{2} \sum_{s}[B(s)-B(s-1)]^{2} \\
& +\sum_{s} \sum_{i} Q_{i}(s)\left\{k_{i}\left[\omega_{i, i} P_{i}(s)+\omega_{i, i-1} P_{i-1}(s)+\omega_{i, i+1} P_{i+1}(s)\right]-P_{i}(s)\right\} \\
& -\sum_{s} \sum_{i} \alpha_{i}(s) P_{i}(s)+\sum_{s} \sum_{i} \gamma_{i}(s) \Psi_{i}(s)
\end{aligned}
$$

where $Q, \alpha$ and $\gamma$ (with subscript $i$ ) are nodewise components of the Lagrange coefficients. $\Psi$ (with subscript $i$ ) is the new xenon concentration in node $i$. Other notation is as in the above equations. The two last terms come from the requirement that the new power densities and xenon concentrations are the same as the old ones, see eqns. (15) and (16).

The second (xenon) sub-system obtains the remaining terms from the Lagrange function, i.e. the terms where the new power density $\Pi(s)$ and old xenon density $\boldsymbol{X}(s)$ appear. The Lagrange function for the xenon sub-system is thus

$$
\begin{aligned}
L_{2}= & \frac{W_{P}}{2} \sum_{s}\left[\frac{1}{N} \sum_{i} \Pi_{i}(s)-P_{d}(s)\right]^{2}+\frac{W_{D}}{2} \sum_{s} \sum_{i}\left[\Pi_{i}(s)-P_{d i}(s)\right]^{2} \\
& +\sum_{s} \sum_{i} Y_{i}(s)\left[X_{i}(s-1) D_{X 0}+I_{i}(s-1) D_{I 0}+\cdots-X_{i}(s)\right] \\
& +\sum_{s} \sum_{i} H_{i}(s)\left[I_{i}(s-1) A_{I 0}+\Pi_{i}(s-1) A_{P 0}+\Pi_{i}(s) A_{P 1}-I_{i}(s)\right] \\
& +\sum_{s} \sum_{i} \alpha_{i}(s) \Pi_{i}(s)-\sum_{s} \sum_{i} \gamma_{i}(s) X_{i}(s)
\end{aligned}
$$

where $Y, H, \alpha$ and $\gamma$ are the components of the Lagrange coefficients. Other notation is as in the above equations. The third term (with $Y$ and $X$ etc.) corresponds to the xenon dynamics eqn. (9). It is not written out completely. The missing parts are the same as in eqn. (9), with $P$ replaced by $\Pi$ and $\operatorname{step} s$ with step $s-1$. The two last terms correspond again to the new constraints, eqns. (15) and (16).

The proper constraints for the rod insertion, eqn. (12), and the coolant temperature deviation, eqn. (13) are attached to the power sub-system while the constraint for the power density, eqn. (14), is attached to the xenon sub-system. Thus both sub-systems contain constraints only for the control, or decision variables. (It should be remembered that the power density $\Pi(s)$ is treated as a control variable in the xenon sub-system.) The treatment of these constraints is discussed together with the solution method. 


\section{The solution method}

\subsection{Principle}

The fundamental principle of the solution method is the same as in similar optimization problems in general: A stationary point of the total Lagrange function (i.e. of $L_{1}+L_{2}$ ) must be found. In practice this means that a point must be found where the partial derivatives of the Lagrangian with respect to all variables are zero. This does not guarantee a global minimum. In fact it is quite possible that the problem has several local minima, mainly because of the strong non-linear effect of the control rod: the power density is changed most in the nodes, where the rod tip moves.

Since the two Lagrange functions $L_{1}$ and $L_{2}$ have only $\alpha(s)$ and $\gamma(s)$ in common, most of the derivatives can be taken separately either on $L_{1}$ or on $L_{2}$. Only derivatives with respect to $\alpha$ and $\gamma$ must be taken on the total Lagrange function.

A two level iterative scheme is to be used as follows: On the higher level the Lagrange coefficients $\alpha(s)$ and $\gamma(s)$ are solved for all nodes and time steps. These are then kept constant at the lower level. They could thus be called the coordination coefficients. In the xenon subsystem the power density (II), xenon $(X)$ and iodine concentrations and the corresponding Lagrange coefficients $\boldsymbol{Y}$ and $\boldsymbol{H}$ are solved. This requires a number of iterations. In the power sub-system the boron concentration, the rod position and the coolant temperature deviation are then solved. The core neutronics model (eqns. (3) to (7)) is used to calculate the power density $\boldsymbol{P}$ in the power sub-system. In that calculation the xenon density obtained in the xenon subsystem is used. Thus the constraint $\boldsymbol{X}=\boldsymbol{\Psi}$ of eqn. (16) is used implicitly. The Lagrange coefficient $Q$ for power is not solved explicitly, it is eliminated analytically. This iterative procedure is repeated until it converges.

The different phases of the solution are now discussed in some more detail. The handling of the constraints for power density and controllers is also described.

\subsection{The coordination coefficients}

The coordination coefficients ( $\alpha$ for power and $\gamma$ for xenon) are solved at the higher level. The solution method is partly based on the solution method of the lower level variables, described below.

\subsection{Coordination coefficient for power $(\alpha)$}

Taking the derivative of the total Lagrange function with respect to the components of the coordination coefficient for power $(\alpha)$ gives

$$
\frac{\partial L}{\partial \alpha_{i}(s)}=\Pi_{i}(s)-P_{i}(s)
$$

where $L$ is the total Lagrange function, $L=L_{1}+L_{2}$. Other notation is as in the equations above. For the converged solution this derivative must be zero. This suggests a gradient method for updating $\alpha(s)$ : at each iteration the change in $\alpha(s)$ is proportional to the difference of the power densities $\Pi(s)$ and $P(s)$. However, such a method might converge slowly, partly because the effect of changing $\alpha(s)$ is not included. 
A faster convergence can be expected, when the anticipated changes in $\Pi(s)$ and $\boldsymbol{P}(s)$ (at the next iteration due to changed $\alpha(s)$ ) are included in eqn. (19), i.e. it is required, that

$$
\Pi(s)+\Delta \Pi(s)-\boldsymbol{P}(s)-\Delta \boldsymbol{P}(s)=0
$$

for all nodes and time steps. The changes $\Delta \Pi(s)$ and $\Delta \boldsymbol{P}(s)$ in power densities at next iteration are (largely) caused by a change $\Delta \alpha(s)$ at this iteration. From eqn. (22) it follows that approximately

$$
\Delta \Pi(s)=-A^{-1} \Delta \alpha(s)
$$

where $A$ is the coefficient matrix defined by eqn. (23).

The change $\Delta \boldsymbol{P}(s)$ can be roughly calculated using the linear model, eqn. (8), when the changes in the boron concentration, the rod position and the coolant temperature are known. The change in the boron concentration at the next iteration can be approximately calculated from eqn. (25)

$$
\left.\Delta B(s)=\frac{1}{W_{B}} Z_{s}^{-1}\left\{M_{b}, \Delta \alpha\right]+\left[\Delta M_{b}, \alpha\right]\right\}
$$

where $Z_{s}^{-1}$ is the row corresponding to time step $s$ for the inverse of the coefficient matrix for solving boron in eqn. (25). $\left[M_{b}, \Delta \alpha\right]$ is a vector with elements

$$
\left[M_{b}, \Delta \alpha\right]_{s}=\sum_{i} M_{b i}(s) \Delta \alpha_{i}(s)=\left\langle M_{b}(s), \Delta \alpha(s)\right\rangle
$$

i.e. the inner products (denoted by $\langle\cdot, \cdot\rangle$ ) of the boron component of the linear model and the change in $\alpha$ for all time steps. The subscript $s$ refers to time step $s$. The second term in brackets is defined similarly. $\Delta M_{b}$ is due to a (possible) change in the linear model.

The control rod position and coolant temperature deviation could be calculated with a simple gradient method, although a more complicated method has been used. For a gradient method it can be argued that the change in the rod position at the next iteration caused by a change in $\alpha$ and the linear model would be

$$
\Delta R(s)=\frac{1}{W_{R}}\left\langle M_{r}(s), \Delta \alpha(s)\right\rangle+\frac{1}{W_{R}}\left\langle\Delta M_{r}(s), \alpha(s)\right\rangle
$$

where the inner product notation is used, i.e. the $\langle\cdot\rangle$ term is again a scalar product. $W_{R}$ is a suitable coefficient. (The solution method is not sensitive to the value of this coefficient.) The last term is again due to a possible change in the linear model.

A similar equation can be written for the coolant temperature deviation. Using all these equations in eqn. (20) finally gives an equation for the change in $\alpha(s)$ at time step $s$, as

$$
\begin{aligned}
A(\Pi-P) & -\frac{1}{W_{B}} A M_{b} Z_{s}^{-1}\left[M_{b}, \Delta \alpha\right]-\frac{1}{W_{B}} A M_{b} Z_{s}^{-1}\left[\Delta M_{b}, \alpha\right] \\
= & \Delta \alpha+\frac{1}{W_{\mathrm{R}}} A M_{r}\left\langle M_{r}, \Delta \alpha\right\rangle \\
& +\frac{1}{W_{\mathrm{R}}} A M_{r}\left\langle\Delta M_{r}, \alpha\right\rangle+\frac{1}{W_{T}} A M_{t}\left\langle M_{t}, \Delta \alpha\right\rangle+\frac{1}{W_{T}} A M_{t}\left\langle\Delta M_{t}, \alpha\right\rangle
\end{aligned}
$$


where the index $s$ for time step has been omitted (except for the s-row of matrix $\left.Z^{-1}\right)$. $W_{T}$ is a suitable coefficient, similar to $W_{R}$.

From the set of linear equations (eqn. (21) for all time steps) $\Delta \alpha(s)$ can be solved for all time steps and all nodes, by first solving the inner products (after multiplying the equations with the linear models). When the inner products are known, eqn. (21) gives $\Delta \alpha(s)$ directly.

\subsubsection{Coordination coefficient for xenon $(\gamma)$}

The condition that the derivative of the Lagrange function with respect to the components of $\gamma$ must be zero has already been used, because of the value of $\boldsymbol{X}(s)$, the xenon concentration of the xenon sub-system is used for $\Psi(s)$, the xenon concentration in the power sub-system. The coordination coefficient $\gamma$ is solved from the requirements that the partial derivatives of the Lagrange function $L_{1}$ of the power subsystem with respect to both the components of the xenon density $\Psi(s)$ and the power density $\boldsymbol{P}(s)$ for each node must be zero. The Lagrange coefficient $\boldsymbol{Q}(s)$ for the power equation is first eliminated from these equations, which then gives a system of linear equations for $\gamma(s)$ in each node. These are to be solved for each time step.

\subsection{The xenon sub-system}

\subsubsection{The main principle}

In the xenon sub-system the power density (I) is taken as the control variable, and the xenon and iodine concentrations as the state variables. This means that the Lagrange function $L_{2}$ of the xenon sub-system corresponds to a rather normal optimization problem. However, since the xenon dynamics equation (eqn. (9)) is nonlinear and the objective function (in $L_{2}$ ) does not contain a quadratic state variable term, only a linear xenon term, the solution cannot be obtained in the usual way, which would lead to the Riccati equation. Instead a quite straightforward iterative method is used.

The requirement that the derivatives of $L_{2}$ with respect to the components of the xenon and iodine concentrations, $\boldsymbol{X}(s)$ and $\boldsymbol{I}(s)$, respectively, are zero results in equations for the Lagrange coefficients $\boldsymbol{Y}(s)$ and $\boldsymbol{H}(s)$. In these equations $\boldsymbol{Y}(s)$ and $\boldsymbol{H}(s)$ (at step $s)$ are connected to $\boldsymbol{Y}(s+1)$ and $\boldsymbol{H}(s+1)($ at step $s+1)$. These equations are solved backwards in time (for each node), keeping the power density and xenon and iodine concentrations constant. The initial condition at $s=K+1$, where $K$ is the last time step is, as usual, $\boldsymbol{Y}(K+1)=\boldsymbol{H}(K+1)=0$.

When the Lagrange coefficients $\boldsymbol{Y}(s)$ and $\boldsymbol{H}(s)$ are known for step $s$, the power density $\Pi(s)$ at step $s$ is solved for all nodes using $\partial L_{2} / \partial \Pi_{i}(s)=0 . X(s), \boldsymbol{I}(s), \boldsymbol{Y}(s)$ and $\boldsymbol{H}(s)$ are kept constant. This is described in more detail below. When $\Pi(s)$ is known, the solution for $\boldsymbol{Y}$ and $\boldsymbol{H}$ then goes to the previous step, i.e. to step $s-1$.

Finally, when the power density is known for all time steps, new xenon and iodine concentrations are calculated (forward in time) for the new power density, using the xenon-iodine equations (eqns. (9) and (10)). This whole procedure is repeated until it converges. In practice the rate of convergence has turned out to be rather fast, in most cases only a few iterations are needed. 


\subsubsection{The solution of the power density}

The derivative of $L_{2}$ with respect to $\Pi_{i}(s)$, the power density in node $i$ at time step $s$ must be zero, as

$$
\begin{aligned}
\frac{\partial L_{2}}{\partial \Pi_{i}(s)}= & \frac{W_{P}}{N}\left[\frac{1}{N} \sum_{j} \Pi_{j}(s)-P_{d}(s)\right]+W_{D}\left[\Pi_{i}(s)-P_{d i}(s)\right] \\
& +Y_{i}(s)\left[D_{P 1}+D_{X P 1} X_{i}(s-1)+\Pi_{i}(s-1) D_{P P}+I_{i}(s-1) D_{P I 1}\right. \\
& \left.+X_{i}(s-1) \Pi_{i}(s-1) D_{X P P}\right] \\
& +Y_{i}(s+1)\left[D_{P 0}+D_{X P 0} X_{i}(s)+2 D_{P 20} \Pi_{i}(s)\right. \\
& +D_{P P} \Pi_{i}(s+1)+D_{P I 0} I_{i}(s) \\
& \left.+2 D_{X P 2} \Pi_{i}(s) X_{i}(s)+D_{X P P} X_{i}(s) \Pi_{i}(s+1)\right] \\
& +H_{i}(s) A_{P 1}+H_{i}(s+1) A_{P 0}+\alpha_{i}(s)=0
\end{aligned}
$$

with the same notation as in equations above. In the third and fourth terms of eqn. (22) (the ' $Y$-terms') the coefficients for the power densities $\Pi(s+1), \Pi(s)$ and $\Pi(s-1)$ are normally small. Old power densities (from the previous iteration) can be used for these terms. Thus the equations for power density are not coupled in time. The coefficient matrix for the resulting set of linear equations for the power densities in all nodes at time step $s$ is simple. The off-diagonal elements are

$$
A_{i j}=W_{P} / N^{2} \quad i \neq j
$$

and the diagonal elements are

$$
A_{i i}=W_{P} / N^{2}+W_{D}
$$

where $A$ denotes the coefficient matrix. $W$ with subscripts $P$ and $D$ are the weights in the objective function for average power density and power density distribution, respectively.

The solution of eqn. (22) is simple, because when the diagonal elements of a matrix are all equal (to $a$ ) and the off-diagonal elements are equal to one, then it can be shown that the diagonal elements of the inverse matrix are

$$
\frac{a+N-2}{a(a+N-2)-N+1} \text { and the others are } \frac{-1}{a(a+N-2)-N+1}
$$

where $N$ is the dimension of the matrix. (In practice the difference $\Pi_{i}(s)-P_{d i}(s)$ is solved from eqn. (22), for numerical reasons.)

\subsubsection{The constraint for power density}

The constraint for power density distribution, eqn. (14) is included in the xenon sub-system, as part of the solution of the power density $\Pi(s)$. If the power density distribution is such that the constraint is satisfied, no special action is taken, and the power density is solved as outlined above. But if it is found at the beginning of the calculation that the constraint is either violated (should not happen) or that the constraint is active, and that the gradient with respect to the power density is such that the constraint is becoming violated, then the calculation is slightly modified. 
The constraint is added to the Lagrange function, as an equivalence constraint, multiplied with a Lagrange coefficient. That coefficient is then eliminated analytically. A slightly modified set of equations results for the power density.

In addition, at the end of each calculation it is checked whether the constraint is violated. If it is, then the power density distribution is modified slightly so that the constraint is merely active (the equality sign is valid).

\subsection{The power sub-system}

Because the Lagrange function of the power sub-system, eqn. (17) corresponds to a quite normal minimization (optimization) problem, it can be transformed to an equivalent but simpler form. The equation, or constraint for power (the $Q$ term) corresponding to eqn. (3) is replaced with the linear model, eqn. (8). The Lagrange coefficient $Q$ is then eliminated using the condition that the partial derivative of the Lagrangian with respect to the power density $P(s)$ must be zero. (Though this coefficient is not the same as the original one, it is eliminated anyway.) The result is a simpler Lagrange function for the power subsystem:

$$
\begin{aligned}
L_{1}=\frac{W_{B}}{2} \sum_{s}[B(s)-B(s-1)]^{2}-\sum_{s} \sum_{i} \alpha_{i}(s)\left[M_{r i}(s) R(s)\right. & \\
& \left.+M_{t i}(s) T(s)+M_{b i}(s) B(s)\right]+C
\end{aligned}
$$

where $C$ is a constant containing values at the linearization point etc. The subscript $i$ at the linear models $(M)$ refers to node $i$.

\subsubsection{The boron concentration}

The requirement that the derivative of this new Lagrange function with respect to the boron concentration at all steps $s$ is zero leads to a set of linear equations for the boron concentration. The equation corresponding to $\operatorname{step} s$ is

$$
W_{B}[2 B(s)-B(s+1)-B(s-1)]=\left\langle\alpha(s), M_{b}(s)\right\rangle
$$

where again the inner product notation is used. At the last time step the term in brackets on the left hand side is $B(s)-B(s-1)$. Due to the simple tridiagonal coefficient matrix the boron concentration can be readily solved from this set of linear equations.

\subsubsection{The rod position and coolant temperature deviation}

The rod position and coolant temperature deviation are solved together. The requirement that the derivatives of the Lagrangian $L_{1}$ (eqn. (24)) with respect to the rod position and coolant temperature are zero leads to the conditions

$$
\left\langle\alpha(s), M_{r}(s)\right\rangle=\left\langle\alpha(s), M_{t}(s)\right\rangle=0
$$

for each time step. Neither the rod position nor the temperature deviation are included explicitly in these conditions. Thus they must be solved indirectly through their effect on the coordination coefficient $\alpha(s)$. This is accomplished by looking for the rod position and coolant temperature deviation that change $\alpha(s)$ (at next iteration) so that the conditions of eqn. (26) are satisfied. This means that the change 
in $\alpha(s)$ must be such that

$$
\left\langle\Delta \alpha(s), M_{r}\right\rangle=-\left\langle\alpha(s), M_{r}\right\rangle
$$

and

$$
\left\langle\Lambda \alpha(s), M_{t}\right\rangle=-\left\langle\alpha(s), M_{t}\right\rangle
$$

where the right hand sides are calculated using the present value of $\alpha(s)$.

These equations are used in the equation of $\Delta \alpha(s)$, eqn. (21), together with the new power density that will correspond to the new rod position and the coolant temperature deviation (to be solved). The power density is expressed using the linear model, eqn. (8). Any possible changes in the linear models can be neglected, because only a correction to the rod position and temperature are calculated. In this way an equation for $\Delta T$ and $\Delta R$ is obtained for each time step, it is

$$
\begin{aligned}
A(\Pi-P)-\frac{1}{W_{B}} A M_{b} Z_{s}^{-1}\left[M_{b}, \Delta \alpha\right] & -A M_{t} \Delta T-A M_{r} \Delta R \\
= & \Delta \alpha-\frac{1}{W_{R}} A M_{r}\left\langle\alpha, M_{r}\right\rangle-\frac{1}{W_{T}} A M_{t}\left\langle\alpha, M_{t}\right\rangle
\end{aligned}
$$

where again the indication for time step (s) is omitted, except for the row $s$ of matrix $Z^{-1}$. Equation 28 is solved similarly to eqn. (21), by multiplying it with the linear models, eliminating $\Delta T$ and $\Delta R$ (using eqn. (27)) and then solving for the inner products $Z^{-1}[M, \Delta \alpha]$. When they are known, the rod position and coolant temperature can be solved.

\subsubsection{Constraints}

The constraints that the rod position and coolant temperature deviation must be within given limits are included in the solution procedure for $\Delta T$ and $\Delta R$. If the constraints are not active, then the solution proceeds as described above. If at some time step, one (or both) of the variables to be solved is at the limit, and the gradient is such that the value tends to go over the limit, the equation at that time step must be modified. In that case the condition of eqn. (27) cannot be used. It must be replaced with $\Delta R=0$, or $\Delta T=0$. The new condition is then used in eqn. (21), which gives a similar equation as eqn. (28). For example, for the condition $\Delta R=0$ it will be

$$
\begin{aligned}
A(\Pi-P)-\frac{1}{W_{B}} A M_{b} Z_{s}^{-1}\left[M_{b}, \Delta \alpha\right]- & A M_{t} \Delta T \\
& =\Delta \alpha+\frac{1}{W_{R}} A M_{r}\left\langle M_{r}, \Delta \alpha\right\rangle-\frac{1}{W_{T}} A M_{t}\left\langle\alpha, M_{t}\right\rangle
\end{aligned}
$$

\subsection{Convergence}

The solution method converges typically in 10-20 iterations, depending on the number of time steps, the constraints and the stability of the core with respect to xenon oscillations. In some cases, with constraints on the power distribution (eqn. (14)), the convergence was slow. In such cases the control rod tended to oscillate a 
few times up and down, before converging to a particular position. This could be avoided by modifying the calculation of the rod position so that the constraint to the power distribution was taken into account also there, and not only in the xenon sub-system.

Since the core model is discrete, the core being divided into about twenty nodes, it may be that the control rod insertion jumps between two nodes from iteration to iteration. It was found that in such cases it is best to compel the rod insertion to be exactly on the boundary between two nodes. This adds one more constraint to the rod position.

If the core model is clearly unstable with respect to xenon oscillations (the amplitude of the oscillations tends to grow rapidly with time) and if the weight of the power density in the objective function is small, the method of solution which has been described might not converge at all. One possible remedy is to add a constraint for the average power density, i.e. the average power density is required to have an exact desired value. In addition a relaxation coefficient may be needed for the coordination coefficient for xenon $(\gamma)$. This means that the change in $\gamma(s)$ from iteration to iteration is reduced from that obtained using the normal solution method.

\section{An example of the application of the method}

As an example, the control of the core model over a time span of 21 hours was calculated. The power requirement during that period is what one would expect, if the reactor had to adjust power output according to consumption: after one hour at full power, a reduction to half power during one hour, six hours at half power and then back to full power, which is kept for the remaining part of the period, see Fig. 1.

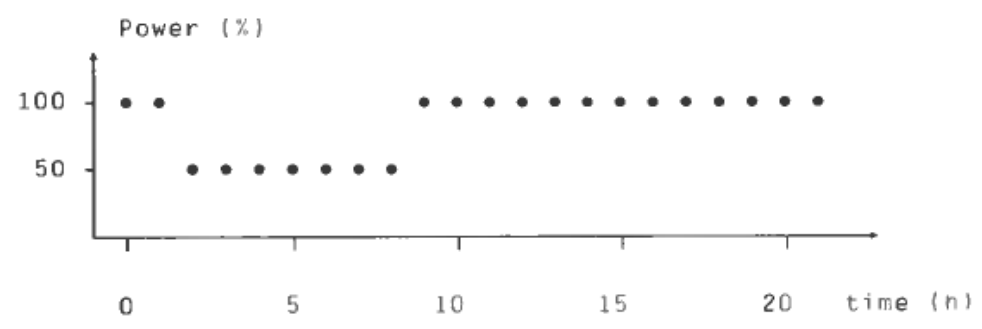

Figure 1. The power requirement in the example.

The core model corresponds to a reactor with a thermal power of about $2400 \mathrm{MW}$. The parameters for the neutronics correspond to a core loaded partly with almost fresh fuel, partly with older fuel, the average burn-up is $16500 \mathrm{MWd} / \mathrm{tU}$. The onedimensional core model was divided into 20 nodes. The time step used is one hour.

The objective function weight for the use of boron is 0.3 , for power density distribution this is 0.1 and 2.0 for the average power density. These values result from experimentation to find suitable values. The power axial offset (difference of power in lower and upper halves of the core divided by the total power) is limited between $\pm 5 \%$, and the coolant temperature deviation between $\pm 5^{\circ} \mathrm{C}$. These limitations are rather realistic, although more restricting limits could also be used in practice. The results are given in Figs. 2-5. 


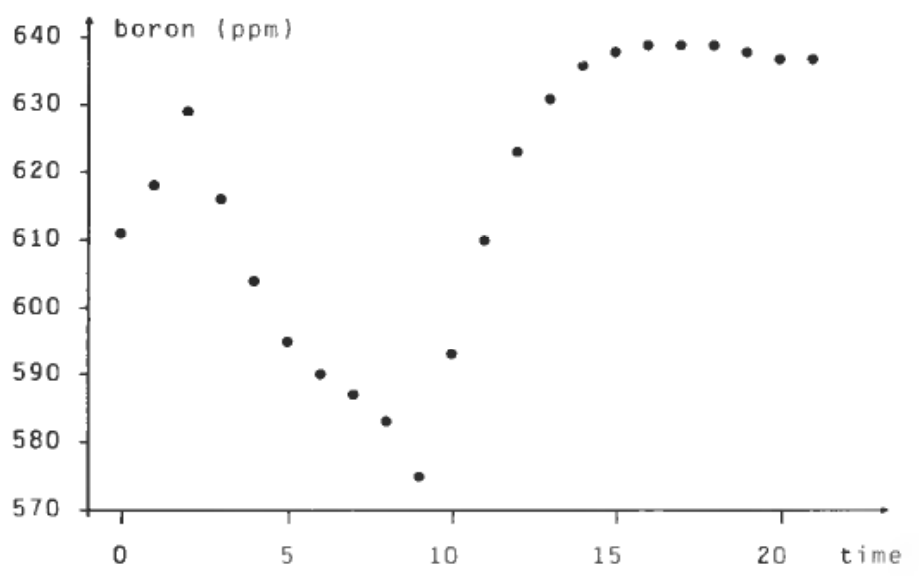

Figure 2. The boron concentration.

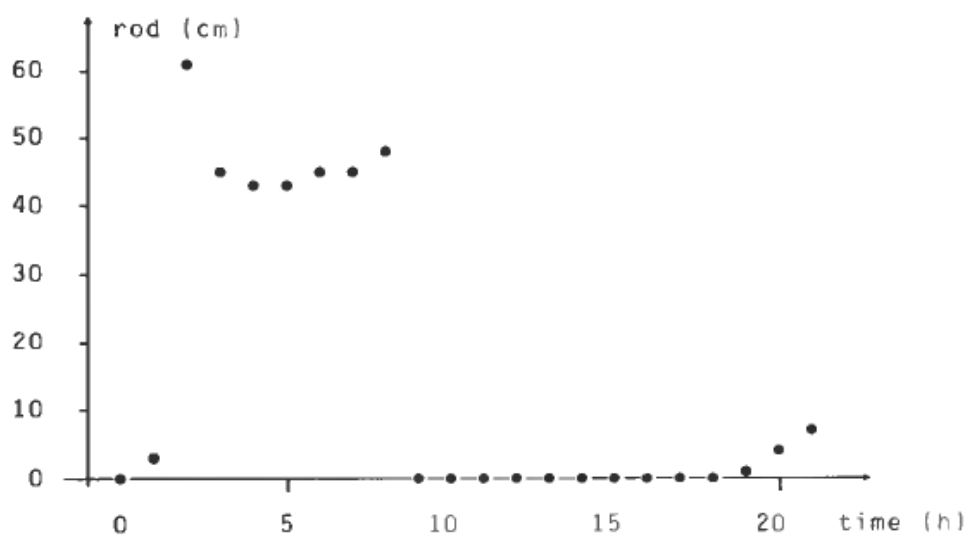

Figure 3. The rod insertion.

It can be seen that the coolant temperature is used actively, and that the changes in the temperature anticipate, or prepare for, the coming transients in power. The movements of the rod mainly seem to attempt to keep the power distribution near to the distribution required, except for the strong insertion when power is reduced. The changes in the boron concentration are smooth, as could be expected. The total

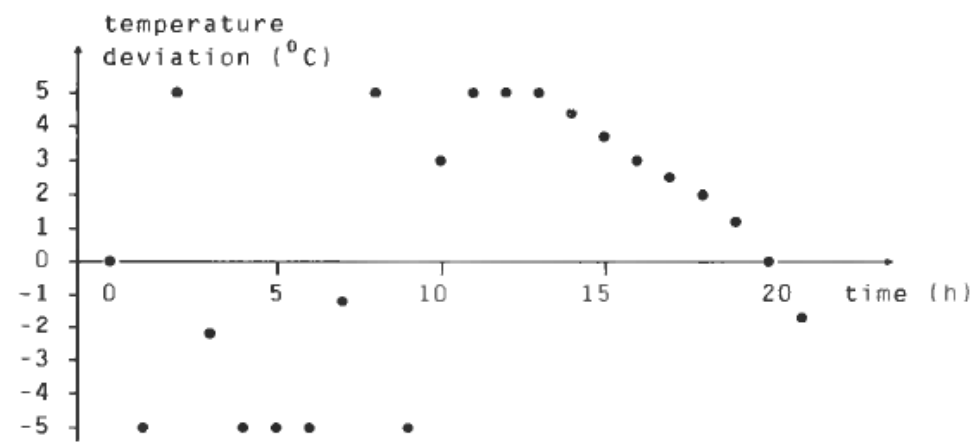

Figure 4. The coolant temperature deviation. 


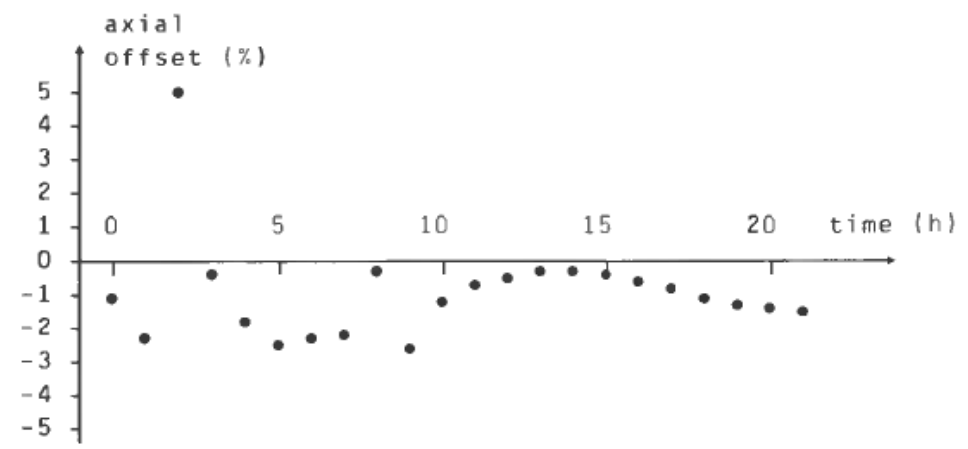

Figure 5. The power axial offset.

power (not shown) follows the required power rather closely, due to the large weight in the objective function. The value of the objective function is 609.6. A total of 24 iterations were needed in order to get the changes in the control rod position below $0.01 \mathrm{~cm}$ from iteration to iteration.

How good, or how optimal is the control which is obtained? First it should be noted that the control is optimal only in the sense of minimizing the value of the objective function with the selected weights. Other weights would result in a different control. Since optimality here means a compromise between different requirements (use of boron, power density distribution etc.), comparisons with some other control sequences might help in assessing the quality of the control obtained. In the following examples the weights in the objective function are the same as in the above example.

In real reactors the coolant temperature is not normally used as a controller, and the power axial offset is strongly limited, typically between $\pm 1 \cdot 5 \%$. If a control of this type is applied to the one-dimensional model discussed here, a control sequence results, where the difference between the boron concentration at the power reduction and increase phases (at 2 hour and 9 hour points) is $64 \mathrm{ppm}$, whereas in the first case it was $54 \mathrm{ppm}$, but the power distribution is nearer to the distribution required. The value of the objective function is 994.5 .

Another extreme is obtained, if there is no constraint on the axial offset, and if the coolant temperature is allowed to vary between $\pm 10^{\circ} \mathrm{C}$. In this case the difference in the boron concentration is about $46 \mathrm{ppm}$. The maximum (by absolute value) axial offset is $6.6 \%$, right after the power reduction, but after that it assumes smaller values. The value of the objective function is $479 \cdot 2$.

Thus, it can be seen that the control obtained in the first case and shown in Figs. $2-5$ is a compromise between these two later cases, i.e. it is an optimal one.

\section{Conclusion}

The work reported here indicates that a relatively simple version of the hierarchical method can be applied to the optimal control problem of the pressurized water reactor core. However, the present method still contains some simplifications that must be improved on, before it can be useful in practice. The important constraints on the rate of change of the boron concentration and coolant temperature must also be added. Further, the core model could be more detailed. The possibility 
of using several rod banks or rods in the control is also important. All these changes will complicate the method, which may also affect the convergence properties.

One clear disadvantage of the method described here (and other similar methods) is that the core model, the objective function and the constraints are built into the solution method to a great extent. Thus it is difficult to change any of them, once the computer program has been written for the selected problem and solution method.

\section{ACKNOWLEDGMENT}

I would like to gratefully acknowledge Mr Sverre Hval for his help in preparing the data for the neutronics and further, for his guidance in the use of the CYGNUS core model.

\section{REFERENCES}

Beraha, D., and KARPPINen, J., 1981, Power distribution control by hierarchical optimisation techniques. In Proceedings of the international topics meeting: Advances in mathematical methods for the solution of nuclear engineering problems, Munich, April 27-29, 1981, 331-355.

BERG, Ø., HVAL, S., and JøRGENSEN, U., 1984, The core surveillance system SCORPIO and its validation against measured pressurized water reactor plant data. AtomkernenergieKerntechnik, 45, 271-276.

EBert, D. D., 1982, Practicality of and benefits from the applications of optimal control to pressurized water reactor manoeuvres. Nuclear Technology, 58, 218-232.

GAutIER, A., 1985, Grey mode operation helps French PWRs to load follow. Nuclear Engineering International, 30, 19-21, No. 364.

Glasstone, S., and SESONSKE, A., 1967, Nuclear Reactor Engineering (Van Nostrand Reinhold Company, New York).

HaugSeT, K., and LeikKonen, I., 1980, Nuclear reactor control by multistage mathematical programming. Modeling, Identification and Control, 1, 119-133.

Hval, S., 1982, The core physics simulator CYGNUS, for on-line applications in core surveillance systems. Report HPR 281, OECD Halden Reactor Project.

LOCKE, G., and BLOBEL, B., 1974, Die Entwicklung des Rechenprogrammes LEIWAR für die Core-Auslegung von Leichtwasser-Reaktoren. GKSS 74/I/1, Gesellschaft für Kernenergiewertung in Schiffbau und Schiffart mbH, Geestacht, West Germany.

Meyer, C. E., Mueller, N., Plumier. M., Watts, M., Leroy, R., and Saint-Mard, C., 1982, Reduced temperature return to power demonstration. Nuclear Technology, 59, 14-19.

Sснотт, H., 1984, Xenonschwingungen in Druckwasserreaktoren. Kernenergie, 27, 277-282.

Schulz, E. J., and LeE, J. C., 1980, Time optimal control of spatial xenon oscillations to a generalized target. Nuclear Science and Engineering, 73, 140-152.

Silvennoinen, P., 1976, Reactor Core Fuel Management (Pergamon Press, Oxford).

Singh, M., and TituI, A., 1978, Systems decomposition, optimization and control (Pergamon Press, Oxford). 